\title{
Insertion of Umbilical Cord on Placenta in Hypertensive Mother
}

\author{
Fatima Jomrud Mohol', Md Mahbubul Karim², Aktari Afroze ${ }^{3}$, \\ Md Jawadul Haque ${ }^{4}$, Most. Monzuara Khatun ${ }^{5}$ \\ ${ }^{1}$ Assistant Professor, Department of Anatomy, Kushtia Medical College, Kushtia, Bangladesh; ${ }^{2}$ Professor \& Head, \\ Department of Anatomy, Pabna Medical College, Pabna, Bangladesh; ${ }^{3}$ Professor \& Head, Department of \\ Anatomy, Rajshahi Medical College, Rajshahi, Bangladesh; ${ }^{4}$ Professor \& Head, Department of \\ Community Medicine, Rajshahi Medical College, Rajshahi, Bangladesh; ${ }^{5}$ Associate Professor, \\ Department of Anatomy, Islami Bank Medical College, Rajshahi, Bangladesh
}

[Received: 21 January 2016; Revised: 6 March 2016; Accepted: 11 June 2016; Published: 1 July 2016]

\begin{abstract}
Background: Hypertensive disorders in pregnancy are responsible for significant amount of maternal and perinatal morbidity and mortality. This study was designed to see the association of umbilical cord insretion on placenta in hypertensive mothers. Objective: This purpose of the present stufdy was to find out the site of umbilical cord insertion on placenta in pregnancy associated with hypertension and to correlate it with the weight of the placenta and the condition of the newborn. Methodology: This descriptive cross-sectional study was conducted at the Department of Anatomy, Rajshahi Medical College and Department of Obstetrics \& Gynaecology at Rajshahi Medical College \& Hospital, Bangladesh. Placental weight, diameter, thickness, insertion site of umbilical cord, insertion percentage of the cord and weight of newborn were noted. The site of cord insertion was detected and insertion percentage was calculated with the help of $\mathrm{d} / \mathrm{r} \mathrm{x}$ 100. Results: A total 130 cases were selected for the study, 30 from normal, 33 from mild, 34 from moderate and 33 from severe hypertensive group respectively. The study demonstrated that mild to severe hypertension had smaller placentas with the tendency of deviation of umbilical cord towards the margin for insertion. Newborns of such mothers had low birth weight mostly; few of them had birth asphyxia. Conclusion: This study has been established that the marginal insertion of umbilical cord on placenta is associated with hypertension and severity of hypertension deviates the insertion site towards the margin. [Journal of National Institute of Neurosciences Bangladesh, 2016;2(2): 89-93]
\end{abstract}

Keywords: Placenta; umbilical cord; insertion; hypertension

Correspondence: Dr. Fatima Jomrud Mohol, Assistant Professor, Department of Anatomy, Kushtia Medical College, Kushtia, Bangladesh; Email: fjmohol@gmail.com; Cell no.: +8801711062726

Conflict of interest: I myself beard all financial supports of this research work except a very small amount of money afforded by the Institution Rajshahi Medical College.

Funding agency: This study was not funded by any authority.

Contribution to authors: FJ Mohol, MM Karim, A Afroze, MJ Haque and MM Khatun were involved in protocol preparation, data collection and literature search up to report writing; furthermore FJ Mohol and MM Karim were involved in manuscript preparation as well as manuscript revision and also in statistical analysis.

How to cite this article: Mohol FJ, Karim MM, Afroze A, Haque MJ, Khatun MM. Insertion of Umbilical Cord on Placenta in Hypertensive Mother. J Natl Inst Neurosci Bangladesh 2016;2(2): 89-93

Copyright: (C2016 Mohol et al. Published by Journal of National Institute of Neurosciences Bangladesh. This article is published under the Creative Commons CC BY-NC License (https://creativecommons.org/licenses/by-nc/4.0/). This license permits use, distribution and reproduction in any medium, provided the original work is properly cited, and is not used for commercial purposes.

\section{Introduction}

Placenta is the first platform for life ${ }^{1}$ and umbilical cord is the life line $e^{2}$ inserted on it through which pregnancy is maintained up to term. It is a unique gestational organ that connects the developing foetus to the uterine wall to allow nutrient uptake, waste elimination \& gaseous exchange through the mother's blood su pply ${ }^{3}$. A pregnancy cannot be proceeding without a healthy placenta ${ }^{4}$. Therefore, any complication during pregnancy may affect both the mother and the foetus. Hypertension is such a common complication still now, especially in developing countries like Bangladesh and also many other developed countries. Pregnancy may induce hypertension in normotensive or may aggravate 
this condition in those who are already hypertensive ${ }^{5}$. It may reduce utero-placental circulation causing foetal hypoxia, distress, intra uterine growth retardation, preterm delivery, low birth weight, foetal and neonatal death $^{6}$. Hypertensive disorders in pregnancy are responsible for significant amount of maternal and perinatal morbidity and mortality ${ }^{7}$.

Placenta is the mirror of maternal and foetal status; it reflects the changes due to maternal hypertension. It was observed by Damania et $\mathrm{al}^{4}$ that hypertension causes low birth weight, low placental weight and other placental abnormalities. Dunhill ${ }^{5}$ described the relation between birth weight, placental area and volume in hypertensive mother. Thomson et $\mathrm{al}^{8}$ reported that placental weight and size are directly proportional to the birth weight.

The site of insertion of umbilical cord on placenta is variable. It inserts more commonly centrally or slightly eccentrally on the foetal surface of placenta ${ }^{6}$. The umbilical cord is normally inserted on the placenta at or near the centre. The incidence of cord insertion is $18 \%$ central, $73 \%$ eccentric, $7 \%$ marginal and $1-2 \%$ velamentous ${ }^{9}$. There is much controversy about the marginal insertion. Percival ${ }^{10}$ observed that eccentric insertion of umbilical cord was most common in normal placenta. It was noticed by Shanklin ${ }^{11}$ that velamentous or marginal type of umbilical cord insertion was found in newborn weighing less than 2500 gms. Rath et $\mathrm{al}^{12}$ observed that marginal insertion is more commonly present on placenta of hypertensive mother. The reports on insertion of umbilical cord on placenta especially in hypertensive pregnancy are scanty. Pregnancy complications like hypertension reflect on placenta and umbilical cord insertion is changed towards the margin in a significant way. Uteroplacental blood flow is decreased in pre-eclampsia because there is maternal vasospasm ${ }^{13}$. Reduced uteroplacental blood flow leading to constrict the fetal arteries associated with the changes seen in placenta of pre eclamptic and eclamptic mothers. This purpose of the present study was to find out the site of umbilical cord insertion on placenta in pregnancy associated with hypertension and to correlate it with the weight of the placenta and the condition of the newborn.

\section{Methodology}

This descriptive cross sectional study was done at the Department of Anatomy, Rajshahi Medical College and Department of Obstetrics \& Gynaecology, Rajshahi Medical College \& Hospital, Rajshahi, Bangladesh. This study was done from January 2010 to December 2010 for a period of one (1) year. Admitted hypertensive pregnant mothers in Obstetrics Indoor, $\mathrm{RMCH}$ were considered as study population. Placental weight, diameter, thickness, insertion site of umbilical cord, insertion percentage of the cord and weight of newborn were noted. The minimum distance between the site of insertion of umbilical cord and the placental margin was measured by the metallic scale and denoted as 'd'. As placenta is more or less circular in outline, the mean radius was estimated from the surface and denoted as ' $r$ '. Then the insertion percentage was calculated by $\mathrm{d} / \mathrm{r} \times 100$. Low insertion percentage indicates marginal insertion, while high insertion percentage implies central insertion. Each placenta was placed on the following categories depending upon the insertion percentage like central, eccentric-lateral, eccentric-medial and marginal. The mean birth weight of newborn falling into each placental group and insertion category was noted. All collected data were methodically recorded into a pre-designed data collection form and analyzed by using computer based SPSS 16.0 program and expressed as mean+SD or in frequency or in percentage. The level of significance was expressed in $\mathrm{P}$ value and $\mathrm{P}$ value $<0.05$ was considered as a level of significance.

\section{Results}

A total 130 cases were selected for the study, 30 from normal, 33 from mild, 34 from moderate and 33 from severe hypertensive group respectively. The study was done on 130 pregnant mothers both normotensive (30) and hypertensive (100) group. Age range of the cases was 28 to 40 years with the mean age $24.79+5.06$ years. Majority (87.0\%) of pregnant mothers belongs to $18-28$ years group. $77 \%$ of study group were hypertensive and $23.0 \%$ were normotensive. The mean diastolic blood pressure was $99.12+18.86 \mathrm{~mm}$ of $\mathrm{Hg}$ (Table 1).

Table 1: Distribution of Diastolic Blood Pressure status of mothers

\begin{tabular}{lcc}
\hline DBP $(\mathbf{m m}$ of Hg) & \multicolumn{2}{c}{ Pregnant mother } \\
\hline & Frequency & Percentage \\
\cline { 2 - 3 } Normal 60-89 $(\mathrm{n}=30)$ & 30 & 23 \\
Mild HTN 90-99 $(\mathrm{n}=33)$ & 100 & 77 \\
Modt HTN 100-110 $(\mathrm{n}=34)$ & & \\
Severe HTN $>110(\mathrm{n}=33)$ & & $\mathbf{1 0 0}$ \\
\hline Total & $\mathbf{1 3 0}$ & \\
\hline
\end{tabular}

Mean $+\mathrm{SD}=99.12 \pm 18.86 \mathrm{~mm}$ of $\mathrm{Hg}$; HTN=Hypertension; $\mathrm{DBP}=$ Diastolic Blood Pressure; Modt=Moderate

The insertion of umbilical cord on placenta was central $11.0 \%$, eccentric-lateral $25.0 \%$, eccentric-medial $31.0 \%$ and marginal $33.0 \%$ (Table 2). 
Table 2: Distribution of Umbilical cord insertion on placenta

\begin{tabular}{lcc}
\hline Site of Insertion & Frequency & Frequency \\
\hline Central (76-100) & 14 & 11 \\
Eccentric-medial (51-75) & 32 & 25 \\
Eccentric-lateral (26-50) & 41 & 31 \\
Marginal (0-25) & 43 & 33 \\
\hline Total & $\mathbf{1 3 0}$ & $\mathbf{1 0 0}$ \\
\hline
\end{tabular}

Mean+SD $=33 \pm 23.23$

The mean insertion percentage was $33.22+23.23$. The maximum insertion site was found $47.0 \%$ eccentricmedial in normal, 39\% eccentric-lateral in mild, $41 \%$ marginal in moderate and $48.0 \%$ marginal in severe hypertension respectively (Table 3 ).

Table 3: Distribution of Umbilical Cord Insertion site in different categories Blood Pressure Status of Mothers

\begin{tabular}{lccccc}
\hline BP status & \multicolumn{3}{c}{ Site of Insertion of Umbilical Cord } & Total \\
\cline { 2 - 5 } & \multicolumn{3}{c}{ Central } & Eccentric-M Eccentric-L Marginal & \\
& $\mathbf{( 7 6 - 1 0 0 )}$ & $\mathbf{( 5 1 - 7 5 )}$ & $\mathbf{( 2 6 - 5 0 )}$ & $\mathbf{( 0 - 2 5 )}$ & \\
\hline Normal & $6(20.0 \%)$ & $14(47.0 \%)$ & $6(20.0 \%)$ & $4(13.0 \%)$ & $\mathbf{3 0 ( 1 0 0 . 0 \% )}$ \\
Mild HTN & $4(12.0 \%)$ & $7(21.0 \%)$ & $13(39.0 \%)$ & $9(27.0 \%)$ & $\mathbf{3 3 ( 1 0 0 . 0 \% )}$ \\
Modt HTN & $3(9.0 \%)$ & $7(21.0 \%)$ & $10(29.0 \%)$ & $14(41.0 \%)$ & $\mathbf{3 4 ( 1 0 0 . 0 \% )}$ \\
Severe HTN & $1(3.0 \%)$ & $4(12.0 \%)$ & $12(36.0 \%)$ & $16(48.0 \%)$ & $\mathbf{3 3 ( 1 0 0 . 0 \% )}$ \\
\hline Total & $\mathbf{1 4 ( 1 1 . 0 \% )}$ & $\mathbf{3 2 ( 2 5 . 0 \% )}$ & $\mathbf{4 1 ( 3 1 . 0 \% )}$ & $\mathbf{4 3 ( 3 3 . 0 \% )}$ & $\mathbf{1 3 0 ( 1 0 0 . 0 \% )}$ \\
\hline
\end{tabular}

$\mathrm{L}=$ Lateral, $\mathrm{M}=$ Medial, $\mathrm{U}$ cord=Umbilical cord, $\mathrm{DBP}=$ Diastolic Blood Pressure; HTN=Hypertension; DBP=Diastolic Blood Pressure; Modt=Moderate

The marginal insertion of umbilical cord on placenta is strongly significant $(\mathrm{P} \leq 0.05)$ in relation to birth weight of newborn (Table 4).

Table 4: Correlation between the insertion of umbilical cord on placenta and birth weight of newborn

\begin{tabular}{lcc}
\hline Site of insertion (\%) & Mean wt of newborn & P value \\
Central (76-100) & $0.448 \mathrm{NS}$ \\
Eccentric- medial(51-75) & $2585 \pm 598.43$ gm & $0.301 \mathrm{NS}$ \\
Eccentric- lateral (26-50) & $0.603 \mathrm{NS}$ \\
Marginal (0-25) & $0.014 \mathrm{~S}$ \\
\hline
\end{tabular}

$\mathrm{NS}=$ Not significant, $\mathrm{S}=$ Significant

The marginal insertion was highly significant in relation to Diastolic Blood Pressure especially in severe hypertension $(\mathrm{P}<0.005)$ (Table 5).
Table 5: Correlation between the umbilical cord insertion on placenta and DBP status of mothers

\begin{tabular}{llccc}
\hline $\begin{array}{l}\text { Site of } \\
\text { insertion (\%) }\end{array}$ & DBP status & Frequency & Percentage & P value \\
\hline Central & Normal & 6 & 20 & 0.17 \\
$(76-100)$ & Mild HTN & 4 & 12 & 0.64 \\
& Moderate HTN & 3 & 9 & 0.84 \\
& Severe HTN & 1 & 3 & 0.11 \\
\hline Eccentric- & Normal & 6 & 20 & 0.42 \\
medial & Mild HTN & 6 & 18 & 0.97 \\
$(51-75)$ & Moderate HTN & 5 & 15 & 0.55 \\
& Severe HTN & 5 & 15 & 0.90 \\
\hline Eccentric- & Normal & 14 & 47 & 0.11 \\
lateral & Mild HTN & 10 & 30 & 0.83 \\
$(26-50)$ & Moderate HTN & 9 & 26 & 0.81 \\
& Severe HTN & 8 & 24 & 0.11 \\
\hline Marginal & Normal & 4 & 13 & 0.06 \\
$(0-25)$ & Mild HTN & 13 & 39 & 0.85 \\
& Moderate HTN & 17 & 50 & 0.30 \\
& Severe HTN & 19 & 58 & $0.01 \mathrm{~S}$ \\
\hline
\end{tabular}

S=Significant

There was significant $(\mathrm{p}<0.05)$ relation between the birth weight of newborn and marginal insertion (Table $6)$.

Table 6: Correlations between the Birth Weight of Newborn and Insertion Percentage of Umbilical Cord

\begin{tabular}{lccc}
\hline Insertion percentage & \multicolumn{2}{c}{ Birth Weight of Newborn } & P value \\
\hline & $<2500 \mathrm{gm}$ & $\geq 2500 \mathrm{gm}$ & \\
\cline { 2 - 3 } Central (76-100) & $42(32.3 \%)$ & $88(67.7 \%)$ & $1.00 \mathrm{NS}$ \\
Eccentric- M (51-75) & & & $0.04 \mathrm{~S}$ \\
Eccentric-L (26-50) & & \\
Marginal (0-25) & & \\
\hline
\end{tabular}

NS= Not significant; $\mathrm{S}=$ Significant

\section{Discussion}

This study was done with the object to see the insertion of umbilical cord on placenta in hypertensive mother. This study showed the prevalence of marginal insertion of umbilical cord on placenta was higher in hypertensive mother especially in moderate and severe hypertension. The age distribution of mothers was revealed that the majority $(87.0 \%)$ were in the age group of 18 to 28 years. The age group was higher which indicates that, this age group is more prone to develop hypertension including eclampsia and pre eclampsia during pregnancy in our country. This age group of pregnancy draws special attention to bring them under proper antenatal check up and follow up. 


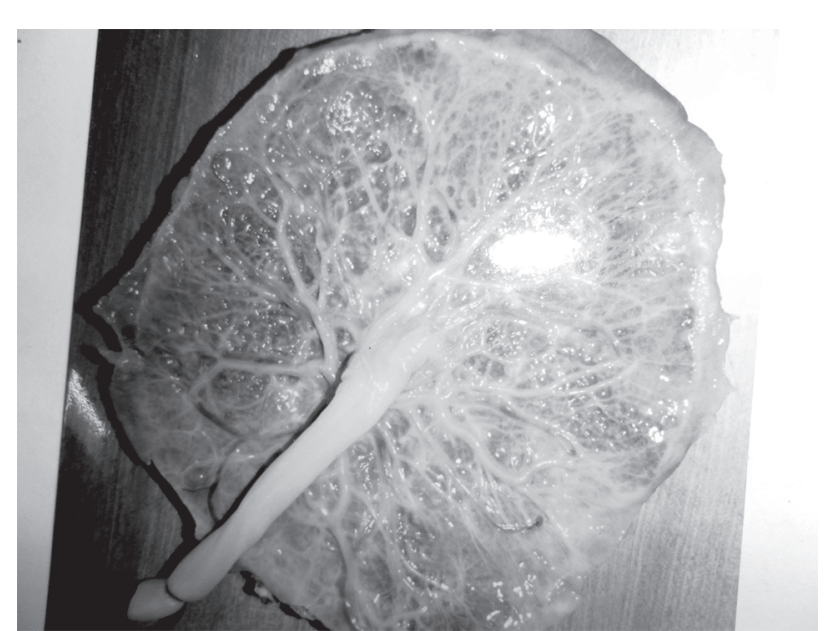

Figure II: Eccentric insertion

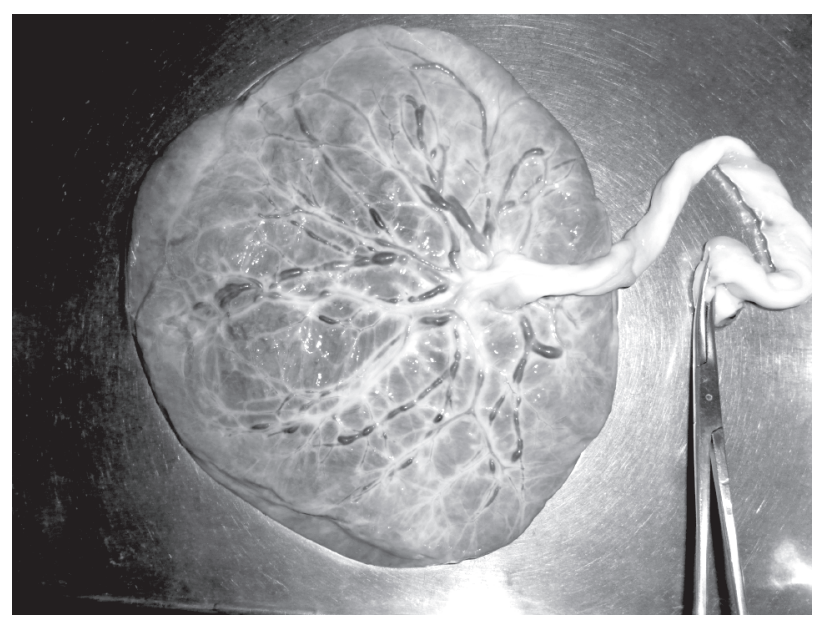

Figure II: Eccentric insertion



Figure III: Marginal insertion

Figure: Site of umbilical cord insertion: Figures of insertion of umbilical cord on placenta central, eccentric and marginal are given below (Figure I, II, III)

The umbilical cord normally inserted on the placenta at or near the centre. In a study, marginal insertion with 0-25 insertion percentage was seen in $13 \%$ cases which were higher by $7 \%$ as noted by Percival ${ }^{10}$. In another study it had been revealed that the marginal insertion with 0 to 25 insertion percentage had a higher score and it was $42.0 \% \%^{12}$. This study showed that in normal, mild and moderate hypertensive cases the findings were $27 \%, 26 \%$ and $26 \%$ respectively. In this study, it has been found that among 130 cases, marginal insertion in normal, mild, moderate and severe hypertension were $13.0 \%, 27.0 \%, 41.0 \%$ and $48.0 \%$ respectively. In moderate and severe hypertension, this score was slightly higher than the previous study. It also showed that marginal insertion was highly significant in relation to weight of newborn $(\mathrm{P}<0.05)$. Low birth weight neonates mostly had low insertion percentage like marginal insertion. The marginal insertion might be responsible for low birth weight babies. Statistically marginal insertion was highly significant in relation to diastolic blood pressure of mother especially in severe hypertension $(\mathrm{P}<0.05)$.

\section{Conclusion}

This study established that the marginal insertion of umbilical cord on placenta was associated with hypertension. Increased severity of hypertension would deviate the insertion site towards the margin. Hypertension in pregnancy might be responsible for low placental weight and low birth weight due to altered vascular pattern. This study showed the method of precise location of umbilical cord insertion by calculating insertion percentage and significant change of insertion site of umbilical cord on placenta. Early detection during antenatal check up by available technique to prevent further risk to mother and fetus by instituting adequate and appropriate measures and to guide health care planning.

\section{References}

1. Anne Cory B, Graham C, Department of Anatomy \& Cell Biology, Queen's University, Stuart Street, Kingston, Ontario, Canada. Placenta-platform for life. Placenta, 2008

2. Brown Teri. Life Cycle of the Placenta. 2010

3. Browne JCM, Veall N. The maternal blood flow in normotensive and hypertensive woman. J Obst Gynaecol British Empire 1953; 60:141-147

4. Damania KR, Salvi VS, Ratnapankhil SK, et al. The Placenta in Hypertensive Disorders of Pregnancy. J Obst Gynaecol India 1989;39:28-31

5. Dunhill MS. Morphometry of Human Placenta, 1966

6. Addai FK, Quashie FJ, Ockleford CD. The mode of insertion of umbilical cord and vessels: association with maternal haemoglobin genotype, neonatal factors, and placental component volumes. Anatomy and embryology. 1994;189(2):107-14

7. Inan Sevinc, Muzaffer Sanci, Deniz Can et al. Comparative 
Morphological Difference between Umbilical cords From Chronic Hypertensive and pre-eclamptic pregnancies. Acta Media Okyama 2002;56(4):177-185

8. Thomson AM, Billewicz WZ, Hytten FE. The Weight of the Placenta in Relation to Birth Weight. J Obst Gynaecol of British Common Wealth 1969;76: 865-872

9. Kliman Harvey J. Yale University School of Medicine. The Umbilical Cord Encyclopedia 2010

10. Percival R. Chorion and Placenta with Placental Ischaemia.14th ed, 1980; The English Language Book Society and
Churchill Livingstone, 30-50

11. Shanklin, DR. The Influence of Placental Lesions in the Newborn Infant. Pedaeatric Clinics of North America 1970; 17:25-42

12. Rath G, Gang K, Sood M. Insertion of Umbilical Cord on Placenta in Hypertensive Mother. J Anatomical Society India 2000;49(2):149-152

13. Landesnon R, Douglas RG, Holze E. The Bulber Conjunctival Vascular Bed in Toxaemias of Pregnancies. Am J Obst Gynaecol 1954;68:170-172 